\title{
Stability of photorefractive spatial solitons
}

\author{
Mordechai Segev, ${ }^{*}$ Bruno Crosignani, ${ }^{+}$Paolo Di Porto, ${ }^{\dagger}$ and Amnon Yariv \\ California Institute of Technology, 128-95, Pasadena, California 91125 \\ Galen Duree, Gregory Salamo, and Edward Sharp ${ }^{*}$ \\ Department of Physics, University of Arkansas, Fayetteville, Arkansas 72701
}

Received February 10, 1994

\begin{abstract}
We present a theoretical analysis of the stability of photorefractive spatial solitons along with experimental results that show that the solitons are stable for small-scale perturbations but break down when the perturbations exhibit a transverse scale comparable with the soliton size (cross section).
\end{abstract}

Self-trapping of optical beams in photorefractive (PR) media occurs when diffraction is exactly balanced by self-scattering of the spatial components of the soliton beam. ${ }^{1,2}$ Our recent observation of the first PR spatial solitons ${ }^{3,4}$ revealed, among a variety of other properties (such as independence of the absolute light intensity and self-trapping in both transverse dimensions), that the PR soliton is stable and may be observed despite index inhomogeneities that are always present in PR materials. Furthermore, we observed that the PR soliton is capable of evolving from an arbitrary input waveform (Fig. 4 of Ref. 3).

In this Letter we provide a theoretical stability analysis and show experimental results that illustrate that the soliton is indeed stable under smallscale perturbations but breaks down when the index perturbations are of a transverse scale comparable with the soliton size. The evolution property of the PR solitons may be viewed as another manifestation of their stability, but, since the evolution stage encompasses other properties (such as modulation instability), we here study stability alone.

We recall the basics of our model ${ }^{1,2}$ and point out that our analysis is restricted to two (transverse $x$ and longitudinal $z$ ) dimensions and cannot explain trapping in two transverse dimensions. Elsewhere ${ }^{5}$ we showed experimentally that the self-trapping effects in the direction parallel to the external electric field ( $x$ direction) exist regardless of the size of the beam in the other transverse dimension $(y)$. Selftrapping in the $y$ direction (perpendicular to the field), however, is generated by tilted gratings and fully depends on the finite extent of the beam in $x$. In this spirit, we demonstrated in Ref. 5 that sheet solitons (of a single transverse dimension) exist for trapping along $x$ but not along $y$ and concluded that trapping in $x$ is independent of trapping in $y$ but not vice versa. A full theoretical model for trapping in both transverse dimensions is under investigation. We therefore restrict our stability analysis to perturbations in this single transverse direction. Fortunately, in most ferroelectric PR crystals inhomogeneities consist primarily of index variations parallel to the crystalline $c$ axis, which is also the growth direction and our transverse axis $x$. This permits experimental study of the soliton stability to perturbations in one transverse dimension.

We start by recalling the paraxial nonlinear wave equation

$$
\left(\frac{\partial}{\partial z}-\frac{i}{2 k} \frac{\partial^{2}}{\partial x^{2}}\right) A(x, z)=\frac{i k}{n_{1}} \delta n(x, z) A(x, z),
$$

where $A$ is the slowly varying complex amplitude of the beam, $k=\omega n_{1} / c$ is the wave number, and $n_{1}$ is the unperturbed index of refraction of the medium. The nonlinear term $\delta n(x, z)$ is obtained by consideration of the mixing process between each pair of plane-wave components:

$$
\begin{aligned}
& \delta n(x, z)=\frac{1}{|A(x, z)|^{2}} \\
& \quad \times \iint A(x-\rho, z) A^{*}\left(x+\rho^{\prime}, z\right) g\left(\rho, \rho^{\prime}\right) \mathrm{d} \rho \mathrm{d} \rho^{\prime},
\end{aligned}
$$

where

$g\left(\rho, \rho^{\prime}\right)=\iint \widehat{\delta n}\left(q_{1}, q_{2}\right) \exp \left[i\left(q_{1} \rho+q_{2} \rho^{\prime}\right)\right] \mathrm{d} q_{1} \mathrm{~d} q_{2}$

is the two-dimensional Fourier transform of the coupling coefficient $\widehat{\delta n}\left(q_{1}, q_{2}\right)$ (which, if multiplied by $-2 \pi i / \lambda$, is identical to $\gamma$, the commonly used expression for the PR coupling coefficient) and $q_{1}$ and $q_{2}$ are the projections of the wave vectors of the plane-wave components in the transverse $(x)$ direction $(0 \leq|q| \leq k)$. The requirement for a spatial soliton,

$$
A(x, z)=U(x) \exp (i \gamma z),
$$

leads to

$$
\gamma-a \frac{U^{\prime \prime}}{U}+b\left(\frac{U^{\prime}}{U}\right)^{2}=0,
$$

where $a=(1 / 2 k)+\left(k / n_{1}\right) I_{20}$ and $b=\left(k / n_{1}\right) I_{11}$, with $I_{11}$ and $I_{20}$ being the real parts of the coefficients of the Taylor expansion of $\widehat{\delta n}\left(q_{1}, q_{2}\right)$, as explained in Ref. 2 . 
The solutions of Eq. (5) are of two types. The first, obtained when $a \neq b$, is

$$
U(x)=U_{0}[\operatorname{sech}(\alpha x)]^{D},
$$

where $D=a /(a-b)$ and the soliton propagation constant is $\gamma=\left[a^{2} /(a-b)\right] \alpha^{2}$. Boundary conditions of $U \rightarrow 0$, when $x \rightarrow \pm \infty$, imply that $D>0$, which furnishes a range for the sign and the amplitude of the external field. ${ }^{2}$ The second type of solution, obtained for $a=b$, is

$$
U(x)=U_{0} \exp \left(-\alpha^{2} x^{2}\right),
$$

with $\gamma=-2 b \alpha^{2}$. In both cases $U_{0}$ is arbitrary, manifesting the independence of absolute light intensity. This property emphasizes that absorption does not affect the solutions, ${ }^{1,2}$ and we therefore expect that the stability will also not be affected.

Let us assume the presence of some material index perturbation giving rise to a deviation from these ideal solutions and examine whether the consequent perturbation in the field amplitude grows or is confined to a small propagation distance. If the PR light-induced index perturbation is real (excluding absorption gratings but permitting uniform absorption $\left.{ }^{1,2}\right)$, i.e., $\delta n(x, z)=\delta n^{*}(x, z)$, one obtains, by multiplying Eq. (1) by $A^{*}$ and adding to its complex-conjugate equation,

$$
\frac{\partial}{\partial z}\left(A A^{*}\right)+\frac{i}{2 k}\left(A \frac{\partial^{2} A^{*}}{\partial x^{2}}-A^{*} \frac{\partial^{2} A}{\partial x^{2}}\right)=0,
$$

which is equivalent to conservation of energy. Introducing a deviation $U^{(1)}(x, z)$ from the soliton solution $U^{(0)}(x) \exp (i \gamma z)$ [where $U^{(0)}$ is one of the two types described above], we write

$$
A(x, z)=U^{(0)}(x) \exp (i \gamma z)+U^{(1)}(x, z),
$$

where $\left|U^{(0)}\right|^{2} \gg\left|U^{(1)}\right|^{2}$ for all $x$ and $z$. Substituting $A(x, z)$ into Eq. (8) and neglecting small terms of the order of $\left|U^{(1)}\right|^{2}$ yields

$$
\begin{gathered}
\left\{U^{(0)}\left[U_{z}^{(1) *}+i \gamma U^{(1) *}\right]+\frac{i}{2 k}\left[U^{(0)} U_{x x}^{(1) *}-U^{(1) *} U_{x x}^{(0)}\right]\right\} \\
\times \exp (i \gamma z)+\left\{U^{(0)}\left[U_{z}^{(1)}-i \gamma U^{(1)}\right]\right. \\
\left.+\frac{i}{2 k}\left[U^{(1)} U_{x x}^{(0)}-U^{(0)} U_{x x}^{(1)}\right]\right\} \exp (-i \gamma z)=0
\end{gathered}
$$

where the subscripts stand for partial $x$ or $z$ derivatives. Grouping terms with identical exponential arguments, which corresponds to considering distances $z \gg 1 / \gamma$ (in practice, $z$ is of the order of $100 \lambda$ or larger), we get

$$
U_{z}^{(1)}-i \gamma U^{(1)}+(i / 2 k)\left\{\left[U^{(1)} U_{x x}^{(0)} / U^{(0)}\right]-U_{x x}^{(1)}\right\}=0 .
$$

Let us now assume that the perturbation $U^{(1)}$ takes place on a scale $l_{p}$ much smaller than the soliton size $l \sim 1 / D \alpha\left(l \gg l_{p} \geq \lambda\right)$ (for the PR soliton of the second type, $l \sim 1 / \alpha$ ). Because $U_{x x}^{(0)} / U^{(0)} \approx 1 / l^{2}$, $U_{x x}^{(1)} / U^{(1)} \approx 1 / l_{p}^{2}$, and ${ }^{2} \gamma=D /\left(l^{2} k\right) \approx 1 /\left(l^{2} k\right)$, the second and third terms on the left-hand side of Eq. (11) may be neglected with respect to the first and the fourth terms, and Eq. (11) becomes

$$
U_{z}^{(1)}-(i / 2 k) U_{x x}^{(1)}=0,
$$

which is a diffusion-type equation for the perturbation $U^{(1)}$ only. The longitudinal scale of $U^{(1)}$ may be as small as $10 \lambda$, its minimal value being restricted only by the paraxial approximation. This implies that $U^{(1)}$ propagates (almost) independently of the soliton solution $U^{(0)}$. On the other hand, because Eq. (12) entails the energy conservation relation

$$
\frac{\mathrm{d}}{\mathrm{d} z} \int_{-\infty}^{\infty}\left|U^{(1)}\right| \mathrm{d} x=0,
$$

$U^{(1)}$ remains small in magnitude, separated from $U^{(0)}$, and diffuses (by diffraction) as it propagates away from its point of origin.

Next, we present experimental results that illustrate the stability of the PR solitons. The experimental configuration is nearly identical to the one in Fig. 1 of Ref. 3. The solitons are observed in a quasisteady state, that is, after the gratings are formed but before the external voltage has been screened. Our experimental observations indicate that the typical time for screening is roughly 2 orders of magnitude larger than the soliton formation time. By use of low intensities $\left(\sim 10 \mathrm{~mW} / \mathrm{cm}^{2}\right)$ and complete elimination of beam fanning, ${ }^{6}$ this quasi-steady-state time slot is now significantly longer than in Ref. 3 and exceeds $5-10 \mathrm{~s}$ in our rhodium-doped SBN:60. We use our method ${ }^{3}$ to image the waveforms of the propagating (or soliton) beam at different planes within the PR crystal. Figures 1 and 2 show transverse cross sections (normalized to the maximal amplitudes in each plane) of the propagating beams at various external fields. The minimum waist $2 w_{0}$ of the beam in Figs. 1 and 2 is $\approx 30 \mu \mathrm{m}$, located $\sim 2 \mathrm{~mm}$ before the entrance face of the crystal (at $z=-2 \mathrm{~mm}$ ). The confocal parameter of the beam is $z_{0} \approx 3.1 \mathrm{~mm}$, so diffraction effects become apparent mainly at the last three planes. In Fig. 1 the input beam is launched through a region of small perturbations, and we examine its behavior at zero field (normal diffraction, left column), and with an external field of $400 \mathrm{~V} / \mathrm{cm}$ (soliton, right column), as it propagates through the medium ( $z=5 \mathrm{~mm}$ indicates the exit face). This figure shows that, despite significant index inhomogeneities (apparent mainly at the third and fifth planes from the top in Fig. 1), the PR soliton is rather stable and smooth, and the perturbations in the waveforms diffuse with increasing distance from the plane in which they were generated. The soliton size $l$ is (FWHM) $\approx 30 \mu \mathrm{m}$, and we estimate the perturbation size $l_{p}$ to be $\approx 3 \mu \mathrm{m}$.

The soliton stability breaks down when the scales of the soliton and the perturbation become comparable, i.e., $l \approx l_{p}$. This situation is present in Fig. 2, which illustrates a case in which the input beam is launched directly into a region of a growth striation 


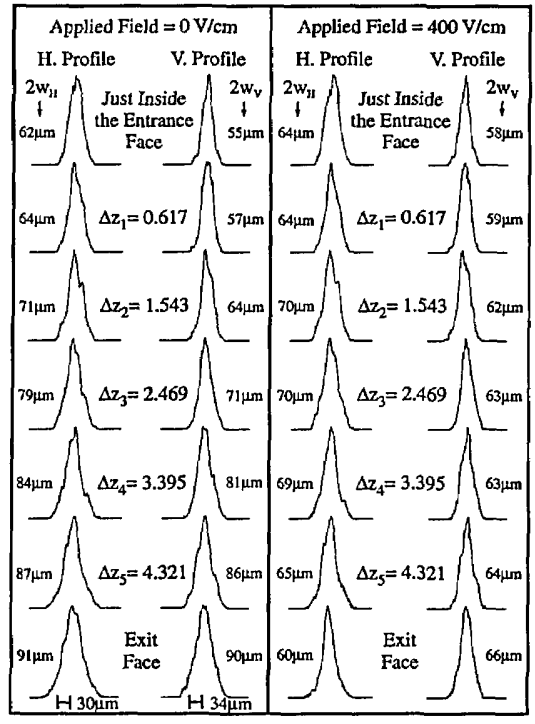

Fig. 1. Horizontal and vertical cross sections of the propagating diffracted beam (zero field, left column) and soliton beam (right column) at various planes (inside the PR crystal) in the presence of small index perturbations. The waveforms are normalized to the maximal amplitudes in each plane. The horizontal and vertical beam spot sizes $\left(w_{H}\right.$ and $\left.w_{V}\right)$ are calculated according to a Gaussian fit.

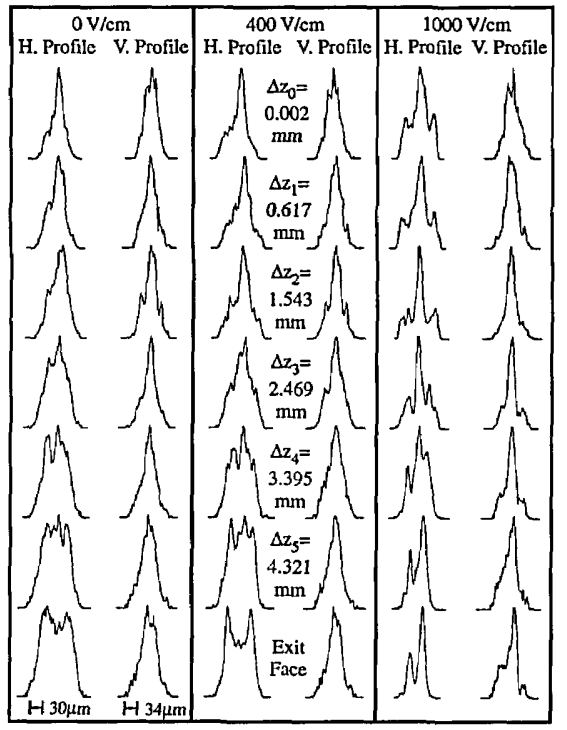

Fig. 2. Horizontal and vertical cross sections of the propagating beam with zero (left column), 400-V/cm (middle column), and $1000-\mathrm{V} / \mathrm{cm}$ (right column) external fields at different planes (inside the PR crystal) in the presence of large index perturbations. The waveforms are normalized to the maximal amplitudes in each plane.

in the same crystal (the striation planes are perpendicular to the crystalline $c$ axis, which is the transverse $x$ axis in our experiment). The left column shows the propagation of the input beam with zero external field at different planes. It is apparent that the large index inhomogeneities not only distort the beam but also significantly increase its diffraction. The middle column shows the propagation with the external field that trapped the beam in the case of Fig. 1. The situation is even more dramatic in the right column, where the external field exceeds the trapping field. The beam breaks down into two filaments, each trying to form its own nondiffracting beam but failing to do so because of their mutual, transversely irregular, interaction (the experiment is also a good indication that soliton beams that are sufficiently close to each other do interact). The soliton size $l$ is, again, $\approx 30 \mu \mathrm{m}$, but $l_{p}$ is now estimated to be $\approx 10 \mu \mathrm{m}$. We estimate the size and dimensionality of the index perturbation that destroys the soliton by varying the location of the input beam and scanning it in both transverse directions. The $x$ region, over which a soliton is not able to form, is roughly $\approx 10 \mu \mathrm{m}$ wide, which corresponds to $l / l_{p} \approx 1 / 3$. Translation in the $y$ direction, on the other hand, does not change significantly the region of self-trapping, a property that is consistent with the assumption that the dominant index perturbations are along the $x$ direction and more-or-less uniform in the $y$ direction.

Our method of imaging different planes of the soliton beam ${ }^{3}$ deserves special attention. Here we note only that, because the material resembles a graded-index waveguide, at least $100 \times 100$ transverse modes can be resolved for a propagation distance of $\approx 1 \mathrm{~cm}$ before imaging becomes impossible as a result of modal dispersion.

*Present address, Department of Electrical Engineering, and Advanced Technology Center for Photonics and Optoelectronic Materials and Princeton Material Institute, Princeton University, Princeton, New Jersey 08544.

${ }^{\dagger}$ Permanent address, Dipartimento di Fisica, Università dell'Aquila, L'Aquila, Italy, and Fondazione Ugo Bordoni, Roma, Italy.

${ }^{\ddagger}$ Permanent address, U.S. Army Research Laboratory, Fort Belvoir, Virginia 22060.

\section{References}

1. M. Segev, B. Crosignani, A. Yariv, and B. Fischer, Phys. Rev. Lett. 68, 923 (1992).

2. B. Crosignani, M. Segev, D. Engin, P. DiPorto, A. Yariv, and G. Salamo, J. Opt. Soc. Am. B 10, 446 (1993).

3. G. Duree, J. L. Shultz, G. Salamo, M. Segev, A. Yariv, B. Crosignani, P. DiPorto, E. Sharp, and R. R. Neurgaonkar, Phys. Rev. Lett. 71, 533 (1993).

4. M. Segev, A. Yariv, G. Salamo, G. Duree, J. Shultz, B. Crosignani, P. DiPorto, and E. Sharp, Opt. Photon. News 4(12), 9 (1993).

5. G. Duree, G. Salamo, M. Segev, A. Yariv, B. Crosignani, P. DiPorto, and E. Sharp, Opt. Lett. 19, 1195 (1994).

6. Because beam fanning is a result of energy-exchange interactions (generated by diffusion fields) between the beam and scattered noise, we use two separate effects to eliminate it: (i) for a beam size that is much smaller than $100 \mu \mathrm{m}$, the cross section for interaction with noise in directions that differ significantly from $z$ is very small [see M. Segev, Y. Ophir, and B. Fischer, Opt. Commun. 77, 265 (1990)]; (ii) we operate at external voltages that gonorato space-charge fields that are much larger than the diffusion field ${ }^{1,2}: E_{0} \gg E_{d} \approx$ $\left(k_{B} T / q\right)[(\partial I / \partial x) / I] \approx\left(k_{B} T / q^{l}\right)$, which is $\approx 8 \mathrm{~V} / \mathrm{cm}$ in our experiments. 